\title{
Simple relation between frustration and transition points in diluted spin glasses
}

\author{
Ryoji Miyazaki $\odot,{ }^{1, *}$ Yuta Kudo, ${ }^{1}$ Masayuki Ohzeki, ${ }^{1,2}$ and Kazuyuki Tanaka ${ }^{1}$ \\ ${ }^{1}$ Graduate School of Information Sciences, Tohoku University, Sendai 980-8579, Japan \\ ${ }^{2}$ Institute of Innovative Research, Tokyo Institute of Technology, Kanagawa 226-8503, Japan
}

(Received 18 January 2020; accepted 9 June 2020; published 15 July 2020)

\begin{abstract}
We investigate a possible relation between frustration and phase-transition points in two-dimensional spin glasses at zero temperature. The relation consists of a condition on the average number of frustrated plaquettes and was reported to provide very good predictions for the critical points at zero temperature, for several twodimensional lattices. Although there has been no proof of the relation, the good correspondence in several lattices suggests the validity of the relation and an important role of frustration in the phase transitions. To examine the relation further, we present a natural extension of the relation to diluted lattices and verify its effectiveness for bond-diluted square lattices. We then confirm that the resulting points are in good agreement with the phasetransition points in a wide range of dilution rate. Our result supports the suggestion from R. Miyazaki [J. Phys. Soc. Jpn. 82, 094001 (2013)] for nondiluted lattices on the importance of frustration to the phase transition of two-dimensional spin glasses at zero temperature.
\end{abstract}

DOI: 10.1103/PhysRevE.102.012135

\section{INTRODUCTION}

Spin glasses have been one of the most attractive subjects in statistical mechanics and have been extensively investigated for decades $[1,2]$. The study of spin glasses, in particular, in infinite dimensions, has developed several elaborate concepts and techniques. For instance, replica symmetry breaking has had a great influence on subsequent studies, e.g., structural glasses $[3,4]$ and information theory $[5,6]$, for revealing their complicated energy landscapes. This success motivates us to tackle a next task, which is to establish theories of more realistic models, namely, finite-dimensional spin glasses. However, it is a very difficult task. This is partially because techniques exploited for the infinite-dimensional models are not as useful in finite dimensions, while it is still difficult to obtain conclusive proofs with numerical simulations.

The gauge transformation [5] has been utilized as a tool for analytically investigating finite-dimensional spin glasses [7-15]. In particular, two-dimensional models have been well investigated using this approach [7,8,10-15]. A consequence of this approach is the conjecture on the vertical boundary of the ferromagnetic phase at lower temperatures than a point in the phase diagram, the so-called Nishimori point [16]. In two dimensions, Ising spin glasses, with increasing the ratio $p$ of antiferromagnetic bonds of spins, undergo the phase transition to the paramagnetic phase at finite temperatures $T$ and to the spin-glass phase at zero temperature [17], as sketched

\footnotetext{
*Present address: System Platform Research Laboratories, NEC Corporation, Tsukuba, Ibaraki 305-8501, Japan.

Published by the American Physical Society under the terms of the Creative Commons Attribution 4.0 International license. Further distribution of this work must maintain attribution to the author(s) and the published article's title, journal citation, and DOI.
}

in Fig. 1. The conjecture states that this boundary of the ferromagnetic phase at lower temperatures than the Nishimori point does not depend on temperature, but is determined only by geometrical properties. In other words, this phase boundary is a vertical line in the $p-T$ plane, as the dashed line in Fig. 1 shows. This conjecture was denied by subsequent detailed studies [10,17-24]. The established phase boundary, however, is almost vertical. This fact implies that geometrical properties take a primary role for the phase transition, even though the conjectured relation does not exactly hold. Note that the importance of revealing the property of this phase transition is not limited in the study of spin glasses. It can influence the study of quantum computation. Indeed, this phase boundary can be interpreted to give the error-correction threshold for topological quantum error-correction codes [25,26].

One of the authors focused on this phase transition, observed with varying $p$, in two-dimensional spin glasses at zero temperature. He reported a possible relation between a geometrical property and this phase transition without utilizing the gauge transformation [27]. The relation is represented as a condition on a quantity concerning frustration [28-30] in the lattice. The condition gives a very close point to the phase-transition point. The good correspondence is found in several two-dimensional lattices and hierarchical lattices [31]. Moreover, the condition for the Sherrington-Kirkpatrick model [32] exactly gives the replica symmetry solution for its transition point at zero temperature. Unfortunately, we have no proof that this agreement is not just an accidental one. The above instances, however, allow us to expect some important role of frustration in the phase transition.

Recently, some of the authors extended this argument to bond-diluted lattices [33]. Their method mainly follows the above one for the nondiluted lattices. Resulting points from their method qualitatively agree with the correct phasetransition points. However, the method was not exactly executed. They instead used an additional ansatz to complete the 


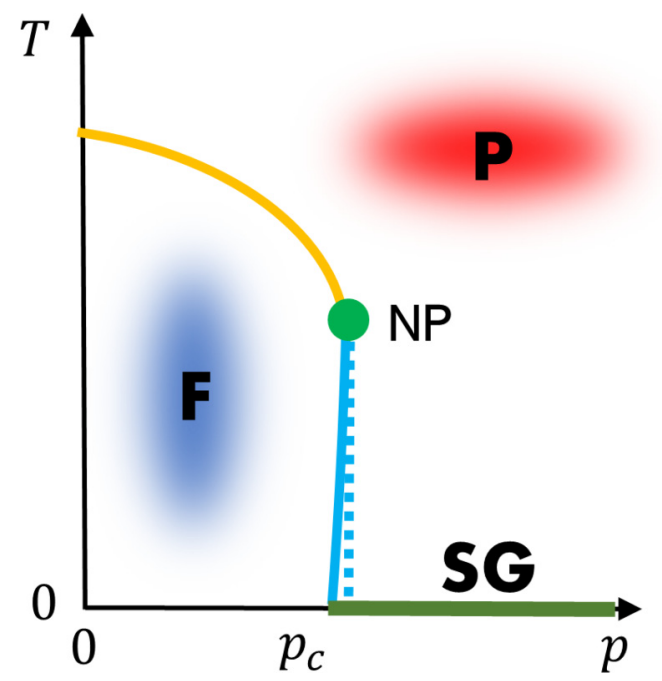

FIG. 1. Schematic phase diagram of $\pm J$ Ising spin glass in two dimensions in the $p-T$ plane, where $p$ and $T$ denote the ratio of antiferromagnetic bonds and temperature, respectively. The system has paramagnetic $(\mathrm{P})$, ferromagnetic $(\mathrm{F})$, and spin-glass $(\mathrm{SG})$ phases. The spin-glass phase lies only at zero temperature. The critical ratio at zero temperature is denoted by $p_{c}$. The vertical dashed line represents the conjectured boundary of the ferromagnetic phase at lower temperatures than the Nishimori point (NP) [16], which deviates from the correct boundary shown by the solid curve.

calculations because of the difficulty due to the inhomogeneity in the diluted lattices. A natural question is whether the good correspondence is also found by the canonical extension without the ansatz or is just caused by the ansatz.

In this paper, we examine the natural extension of the method for nondiluted lattices [27] to bond-diluted lattices in two dimensions without any extra ansatz. The next section gives the prescription of the method. We describe two natural ways of extension of the method for nondiluted lattices to the diluted case. The effectiveness of the method is verified for the diluted square lattice in Sec. III. We apply the method with perturbative analysis expanded from the nondiluted case and numerical calculations. The obtained points are compared with the correct phase-transition points. We summarize and discuss our results in Sec. IV.

\section{PRESCRIPTION}

We investigate $\pm J$ Ising spin glass [5], defined by

$$
H=-\sum_{\langle i, j\rangle} J_{i j} \sigma_{i} \sigma_{j}
$$

where $\langle i, j\rangle$ denotes a pair of nearest-neighbor sites on a lattice. The coupling constants $J_{i j}$ are independently and identically distributed according to the distribution $P\left(J_{i j}\right)=$ $p \delta\left(J_{i j}+J\right)+(1-p) \delta\left(J_{i j}-J\right)$ with $J>0$, where $\delta(\cdot)$ is the Dirac delta function. Note that we define $p$ as the probability that a bond of spins is an antiferromagnetic one. Ising spin $\sigma_{i}$ takes 1 or -1 . This model has been extensively used as an elementary model of spin glasses in finite dimensions [5]. We only consider the model on two-dimensional lattices.
We focus on frustration for plaquettes [28-30]. A plaquette is an elementary loop of edges on a lattice, which cannot be divided into multiple subloops. For example, a plaquette on a square lattice is a square composed of four edges. When a plaquette has an odd number of antiferromagnetic couplings, there is no spin configuration such that each link in the plaquette gives a contribution to the energy of $-J$. Consequently, there is frustration at the plaquette. Such a plaquette is called frustrated plaquette. The average number of frustrated plaquettes on a lattice over the bond distribution plays a central role in the argument below. That average is calculated as

$$
N_{\text {fra }}(p)=\left\langle\sum_{c} \frac{1}{2}\left(1-\prod_{\langle i, j\rangle \in c} \frac{J_{i j}}{J}\right)\right\rangle_{p}^{\text {af }},
$$

where $c$ are indices for plaquettes, and $\langle\cdot\rangle_{p}^{\text {af }}$ denotes the average over the antiferromagnetic-bond distribution for $p$. The coupling constants are independent of each other, and hence we can rewrite the function as

$$
\begin{gathered}
N_{\text {fra }}(p)=\sum_{n} N_{\text {pla }}^{(n)} f_{n}(p), \\
f_{n}(p)=\frac{1}{2}\left[1-(1-2 p)^{n}\right],
\end{gathered}
$$

where $N_{\text {pla }}^{(n)}$ is the number of plaquettes composed of $n$ edges on the lattice. The function $f_{n}(p)$ gives the probability that a plaquette composed of $n$ edges is frustrated. For the square lattice, the sum reduces to the single term for $n=4$. This expression also concerns lattices with multiple types of plaquettes, e.g., the kagome lattice.

One of the authors focused on the function [27] defined by

$$
v(p)=\frac{d N_{\mathrm{fra}}(p)}{d p}\left(\frac{d N_{\mathrm{af}}(p)}{d p}\right)^{-1} .
$$

Here, $N_{\mathrm{af}}(p)$ is the average number of antiferromagnetic bonds over the bond distribution for $p$, calculated as $N_{\mathrm{af}}(p)=p N_{\text {edg }}$, where $N_{\text {edg }}$ is the number of edges in the lattice. He reported [27] that the condition $v(p)=1$ gives a value of $p$ that is close to the phase-transition point for the model at zero temperature. For instance, the value $p \simeq 0.1031$ yielded from the condition for the square lattice is very close to the actual phase-transition point numerically obtained as $p=0.1033$ (1) [23] or 0.1045(11) [24]. The good correspondence is found in several two-dimensional lattices and hierarchical lattices [31]. Interestingly, the condition for the Sherrington-Kirkpatrick model [32] exactly gives the replica symmetry solution for its phase-transition point at zero temperature.

We extend the above argument to apply to $\pm J$ Ising spin glass on bond-diluted lattices. Each edge in the lattices is absent with probability $q$. The probability that a bond is an antiferromagnetic one thus turns to $(1-q) p$. We introduce two ways of extension to this case. The first one generalizes the function in Eq. (3) as the average number of frustrated plaquettes over diluted lattices as well as the antiferromagneticbond distributions, namely,

$$
N_{\text {fra }}(q, p)=\sum_{n}\left\langle\left. N_{\text {pla }}^{(n)}\right|_{q} ^{\text {di }} f_{n}(p),\right.
$$


where $\langle\cdot\rangle_{q}^{\text {di }}$ denotes the average over the diluted-bond distribution for $q$. We have utilized the fact that $f_{n}(p)$ is not affected by the bond dilution. Accordingly, we define a generalized function of $v(p)$ in Eq. (5) by

$$
v(q, p)=\frac{\partial N_{\mathrm{fra}}(q, p)}{\partial p}\left(\frac{\partial N_{\mathrm{af}}(q, p)}{\partial p}\right)^{-1} .
$$

Here, $N_{\mathrm{af}}(q, p)$ is the average number of antiferromagnetic bonds over the bond distribution for $q$ and $p$, calculated as $N_{\text {af }}(q, p)=(1-q) p N_{\text {edg }}$, where $N_{\text {edg }}$ is the number of edges in the lattice without dilution. The condition $v(p)=1$ is generalized as $v(q, p)=1$ for the bond-diluted lattices. For the other extension, we calculate the average number of frustrated plaquettes and antiferromagnetic bonds over only the antiferromagnetic-bond distribution on a given bond-diluted lattice. Equation (5) then gives the function $v(p)$ for the lattice. We consider a bond-diluted lattice for this extension, whereas we took the average over bond-diluted lattices for $q$ for the first extension. The second extension could give different solutions of $v(p)=1$ for different diluted lattices. We expect to obtain, however, a typical solution from the different diluted lattices due to the self-averaging property [5] of the system. The possible typical one is regarded as the solution obtained from our method for $q$. In addition, we can consider a minor change of this extension in estimation of the typical solution; we estimate the average of the function $v(p)$ over bond-diluted lattices and obtain the solution of $v(p)=1$ for the averaged function instead of the average of solutions themselves over different lattices. Hereafter, we examine whether the two procedures of extension give $p$ close to the correct phase-transition point of the model on diluted lattices for $q$ observed with varying $p$ at zero temperature.

\section{SQUARE LATTICE}

\section{A. Perturbative calculations}

We first restrict our interest to the systems in which the number of lacked edges is small and obtain its expansion in terms of $q$. Motivated by the fact that $p$ for the condition $v(p)=1$ for the square lattice is extremely close to the phasetransition point [27], we analyze the model on the square lattice. Here, we only attempt the first way of extension, where we calculate $v(q, p)$ in Eq. (7), because it is intractable to analytically obtain the solutions with the second extension. The second one will be examined with numerical calculations in Sec. III B. It should be noted that $N_{\text {edg }}$ and $N_{\text {pla }}$ used below denote the numbers of edges and plaquettes, respectively, for the lattice without dilution.

We obtain $N_{\text {fra }}(q, p)$ in Eq. (6) for terms up to $q^{n}$ by considering lattices in which the number of lacked edges is smaller than $n+1$. This is because the probability that a lattice lacks $n$ edges is $q^{n}(1-q)^{N_{\text {edg }}-n}$, and because $\left\langle N_{\text {pla }}^{(n)}\right\rangle_{q}^{\mathrm{di}}$ in $N_{\text {fra }}(q, p)$ is the average number of $n$-edge plaquettes over those lattices. Figure 2 shows examples of square lattices removed 1, 2, or 3 edges. Note that there can exist edges which do not belong to any plaquette and thus do not contribute to frustration. For instance, the edge in the eight-edge square on the lattice shown in Fig. 2(h) does not belong to any loop of edges. As an example of computing $N_{\text {fra }}(q, p)$, let us consider (a)

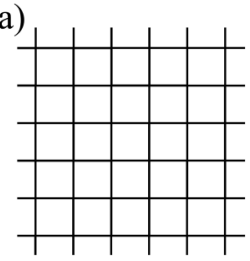

(d)

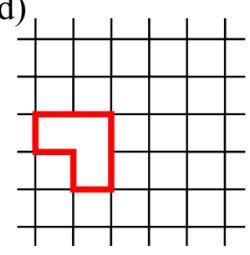

(b)

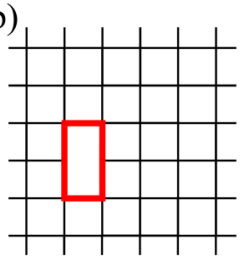

(e)

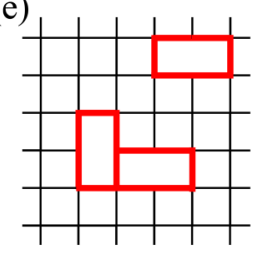

(c)

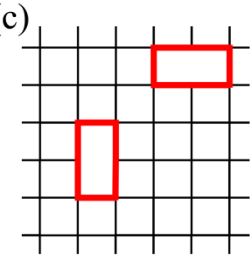

(f)

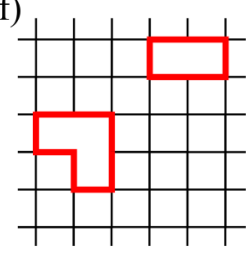

(g)

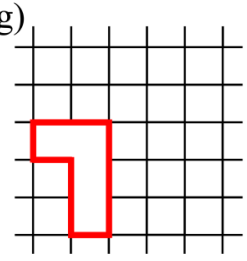

(h)

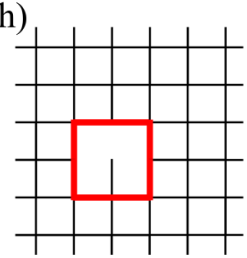

FIG. 2. Examples of square lattices without or with dilutions treated in calculations of $N_{\text {fra }}(q, p)$ for small $q$. Plaquettes, which are not composed of four edges, generated by removing edges are highlighted with thick red lines. (a) The square lattice without dilution. Examples of square lattices lacking (b) an edge, (c), (d) two edges, and (e)-(h) three edges. The examples are distinguished by the number of lacked edges and the number of edges for generated plaquettes.

a lattice lacking an edge as shown in Fig. 2(b). The probability that such a lattice is realized is $q(1-q)^{N_{\text {edg }}-1}$. The number of positions at which an edge is absent is $N_{\text {edg }}$. By removing an edge from the primary square lattice, the number of four-edge plaquettes reduces to $N_{\text {pla }}-2$, while a six-edge plaquette is generated. The contribution of such lattices to $N_{\text {fra }}(q, p)$ is thus $q(1-q)^{N_{\text {edg }}-1} N_{\text {edg }}\left[\left(N_{\text {pla }}-2\right) f_{4}(p)+f_{6}(p)\right]$. Taking into account the lattices removed 1,2 , or 3 edges, we obtain

$$
\begin{aligned}
N_{\text {fra }}( & , p) \\
= & (1-q)^{N_{\text {edg }}} N_{\text {pla }} f_{4}(p) \\
& +q(1-q)^{N_{\text {edg }}-1} N_{\text {edg }}\left[\left(N_{\mathrm{pla}}-2\right) f_{4}(p)+f_{6}(p)\right] \\
& +q^{2}(1-q)^{N_{\text {edg }}-2} N_{\text {edg }} \\
& \times\left\{\frac{N_{\text {edg }}-7}{2}\left[\left(N_{\mathrm{pla}}-4\right) f_{4}(p)+2 f_{6}(p)\right]\right. \\
& \left.+3\left[\left(N_{\mathrm{pla}}-3\right) f_{4}(p)+f_{8}(p)\right]\right\} \\
& +q^{3}(1-q)^{N_{\text {edg }}-3} N_{\mathrm{edg}} \\
& \times\left\{\left[2\left(N_{\mathrm{edg}}-12\right)+14\left(N_{\mathrm{edg}}-13\right)\right.\right. \\
& \left.+\left(N_{\mathrm{edg}}-23\right)\left(N_{\mathrm{edg}}-14\right)\right] \\
& \times \frac{1}{3 !}\left[\left(N_{\mathrm{pla}}-6\right) f_{4}(p)+3 f_{6}(p)\right] \\
& +3\left(N_{\mathrm{edg}}-10\right)\left[\left(N_{\mathrm{pla}}-5\right) f_{4}(p)+f_{6}(p)+f_{8}(p)\right]
\end{aligned}
$$



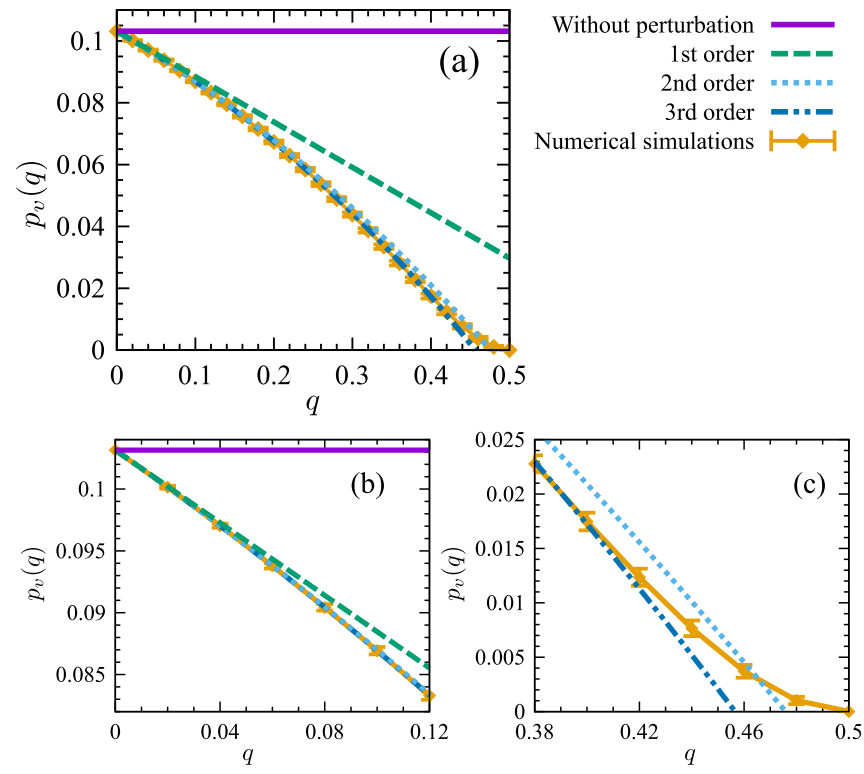

FIG. 3. Solutions $p_{v}(q)$ obtained with the perturbative calculations of the first extension and with the numerical calculations of the second one. The $n$ th-order perturbative solution is a function of $q$ taking into account lower-order terms than $q^{n+1}$ given in Eq. (10) for $n=0$ (without perturbation), 1,2 , and 3 . The numerical simulations are done for the lattices generated by removing edges from the square lattice of $L \times L$ units, where $L$ for the result shown here is 128 . The numerical solutions are estimated by averaging $10^{4}$ instances. (a) The solutions for $0 \leqslant q \leqslant 0.5$. Closeups of the regions near (b) $q=0$ and (c) $q=0.5$.

$$
\begin{aligned}
& +9\left[\left(N_{\text {pla }}-4\right) f_{4}(p)+f_{10}(p)\right] \\
& \left.+2\left[\left(N_{\text {pla }}-4\right) f_{4}(p)+f_{8}(p)\right]\right\}+O\left(q^{4}\right) \\
= & N_{\text {pla }} f_{4}(p)+N_{\text {edg }}\left[-2 f_{4}(p)+f_{6}(p)\right] q \\
& +3 N_{\text {edg }}\left[f_{4}(p)-2 f_{6}(p)+f_{8}(p)\right] q^{2} \\
& +N_{\text {edg }}\left[-2 f_{4}(p)+15 f_{6}(p)-22 f_{8}(p)+9 f_{10}(p)\right] q^{3} \\
& +O\left(q^{4}\right) .
\end{aligned}
$$

Substituting this into Eq. (7), we then have

$$
\begin{aligned}
v(q, p)= & 2 r^{3}\left[1-3\left(1-r^{2}\right) q+3\left(1-5 r^{2}+4 r^{4}\right) q^{2}\right. \\
& \left.+\left(-1+30 r^{2}-76 r^{4}+45 r^{6}\right) q^{3}\right]+O\left(q^{4}\right),
\end{aligned}
$$

where $r=1-2 p$. We have used a relation $N_{\text {pla }}=N_{\text {edg }} / 2$ for the square lattice. The solution $p_{v}(q)$ of $v(q, p)=1$ for $q$ is expanded in terms of $q$ as

$$
\begin{aligned}
p_{v}(q)= & \frac{1}{2}-2^{-4 / 3}-\left[2^{-4 / 3}-2^{-2}\right] q-\left[2^{-4 / 3}-\frac{1}{4}\right] q^{2} \\
& -\left[\frac{11}{6} \times 2^{-4 / 3}-\frac{4}{3} \times 2^{-5 / 3}-\frac{1}{4}\right] q^{3}+O\left(q^{4}\right) .
\end{aligned}
$$

The solutions containing the terms up to $q^{n}$ for $n=0,1,2$, and 3 are drawn in Fig. 3, where a result of the second extension given in Sec. III B is also shown for comparison.

\section{B. Numerical calculations}

We run numerical simulations of the second extension, where the solution of $v(p)=1$ for each bond-diluted lattice

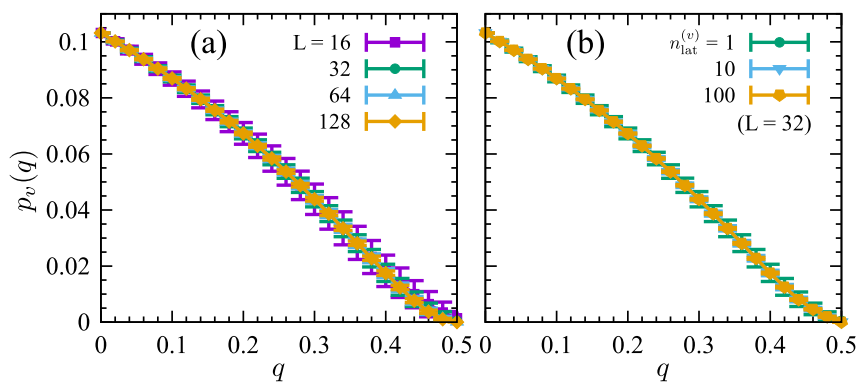

FIG. 4. Solutions $p_{v}(q)$ of $v(p)=1$ estimated from $10^{4}$ instances of numerical simulations based on the second extension. (a) The solutions for $L=16,32,64$, and 128. (b) The solutions for the averaged function over $n_{\text {lat }}^{(v)}$ lattices for $n_{\text {lat }}^{(v)}=1,10$, and 100 for $L=32$.

is estimated. We first generate a bond-diluted lattice under the periodic boundary condition in which an edge is lacked with probability $q$ and then count plaquettes and edges. We do not consider whether bonds in the lattice are ferromagnetic ones or antiferromagnetic ones, since this matter concerns only the $p$ dependence of $N_{\text {fra }}(p)$ that is already determined by $f_{n}(p)$ given in Eq. (4). We then obtain the function $v(p)$ and the solution of $v(p)=1$ for the lattice. Sampling solutions for a number of diluted lattices for $q$ in this way, we estimate the average and variance of the solutions. The average is also denoted by $p_{v}(q)$ for simplicity.

Figure 3(a) shows the plot of the estimated solutions as a function of $q$ for $0 \leqslant q \leqslant 0.5$. The perturbative solutions based on the first extension are also displayed for comparison. The square lattice before the dilution has $L \times L$ units (squares), where $L$ for the result shown in Fig. 3 is 128 . Solutions of $v(p)=1$ are sampled from $10^{4}$ lattices generated from the distribution for $q$. The variance of the solutions over different lattices is small. The averaged value is thus regarded as the probable solution for $q$ obtained with the second extension of our method. In addition, the obtained numerical solution of the second extension is in good agreement with the perturbative solutions of the first extension for small $q$ [Fig. 3(b)]. In particular, the numerical solution and the third-order perturbative solution show good correspondence for $q \leqslant 0.4$. This result demonstrates that both of the ways of extension lead to almost identical solutions. The perturbative solutions, however, do not exhibit the nonconcavity found in the numerical ones at $q>0.4$ [Fig. 3(c)], where the perturbative analysis expanded from $q=0$ would be unreliable. We should remark that the curve of the numerical solutions converges to 0 with $q$ approaching 0.5 [Fig. 3(c)], which agrees with the exact phase-transition point at $q=0.5$.

The size $L$ dependence of the solutions is shown in Fig. 4(a). We find no definite difference in the average of the solutions between investigated $L$ except for $q \geqslant 0.4$, where a slight decrease is observed with increasing $L$. We therefore expect that the finite-size effect of our solutions is small. On the other hand, the variance of the solutions clearly decreases as $L$ increases. As mentioned above, the average of the solutions over bond-diluted lattices agrees well with the solutions of the first extension, where we obtained the solutions with the average number of frustrated plaquettes. This finding 
and the decrease of the variance with increasing $L$ suggest that the small variance of the numerical solution originates from the typicality of the number of frustrated plaquettes that could be involved in the self-averaging property [5] of the system.

Using a rather small lattice $(L=32)$, we also execute the other procedure of estimation of the typical solution mentioned in the end of Sec. II, where we compute the solutions of the averaged $v(p)$ over $n_{\text {lat }}^{(v)}$ lattices. To observe the variance of the resulting solutions, they are sampled $10^{4}$ times. This estimation for $n_{\text {lat }}^{(v)}=1$, hence, corresponds to the above method, the result of which is shown in Fig. 4(a) $(L=32)$. Figure 4(b) displays the average of the obtained solutions with error bars over $10^{4}$ samples for $n_{\text {lat }}^{(v)}=1,10,100$. Increasing $n_{\text {lat }}^{(v)}$ does not make any definite differences in the average of the solutions, but just suppresses the fluctuation of the solutions. Therefore, we use the result for $n_{\text {lat }}^{(v)}=1$ as the solution of our method for the bond-diluted lattices.

\section{Comparison with the minimum-weight perfect-matching algorithm}

We compare the obtained solution of $v(p)=1$ with the correct phase-transition point. The latter has already been estimated in the context of the quantum error correction for the surface code with loss by using the minimum-weight perfectmatching (MWPM) algorithm [34]. We, however, performed the similar calculations in a number of points of $q$ because we need detailed illustration of the $q$ dependence of the critical point. We followed the treatment of the diluted lattices as well as the system size, $L=16,24,32$, and the number of instances of diluted lattices, $5 \times 10^{4}$, in Ref. [34]. For given $q$ and $L$, we estimate a function of $p$, which is referred to as $p_{\text {fail }}$ in Ref. [34], by using $5 \times 10^{4}$ instances of lattices. The function is associated with the phase transition. The obtained functions are scaled according to the finite-size scaling ansatz in Ref. [21]. The collapse of the scaled functions for different $L$ gives the critical point for each $q$ expected in the infinite- $L$ limit. We just estimate the expectation of the critical points and could not explicitly show errors in our analysis.

The average $p_{v}(q)$ of solutions of $v(p)=1$ is in good agreement with the obtained critical values $p_{c}$ in the whole range of $q(0 \leqslant q \leqslant 0.5)$, except for $q \simeq 0.34$, as shown in Fig. 5(a). The obtained two curves for small $q$ are highlighted in Fig. 5(b). The correspondence at $q=0$ previously found [27] is reproduced. Interestingly, the slope of $p_{v}(q)$ at $q=0$ is also very similar to that of $p_{c}$. Moreover, they remain almost identical curves for $q \leqslant 0.14$. This finding demonstrates that our method effectively captures the $q$ dependence of the true phase-transition point, for small $q$ at least. For larger $q$, $p_{v}(q)$ departures from $p_{c}$ and takes a little smaller value. The difference between them takes its maximum around $q \simeq 0.34$, but it is still small. For $q \geqslant 0.34, p_{c}$ decreases more rapidly than $p_{v}(q)$, and they take similar values again at $q \geqslant 0.4$ [Fig. 5(c)]. This agreement is due to the nonconcavity for $q \geqslant 0.4$ in $p_{v}(q)$, highlighted in Figs. 5(c) and 5(d), that also appears in $p_{c}$ [Figs. 5(c) and 5(e)]. Both of the curves finally converge to 0 with $q$ approaching 0.5 [Fig. 5(c)]. This good correspondence in the range of $q$ implies a scenario that our
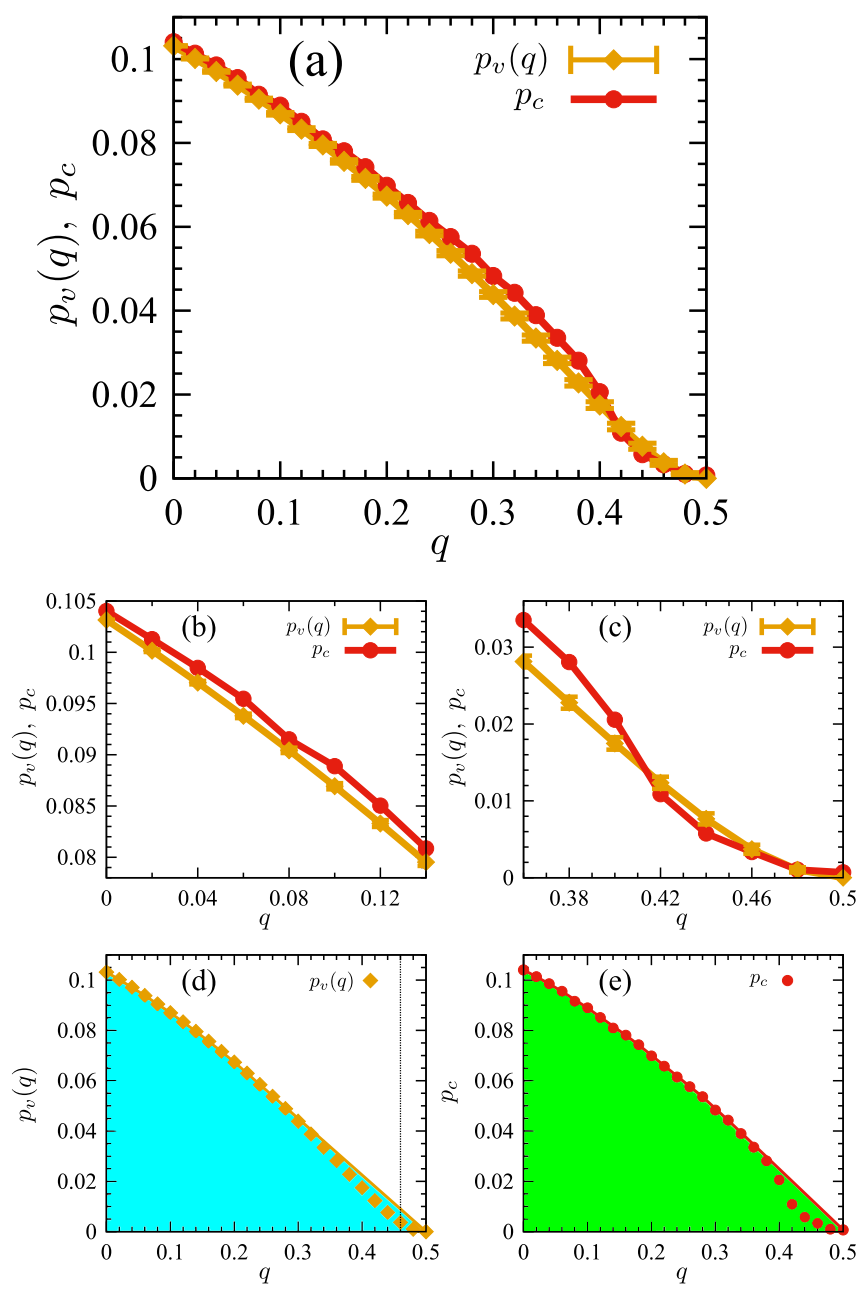

FIG. 5. Solutions $p_{v}(q)$ of $v(p)=1$ for $L=128$ (the same one as shown in Fig. 3) and the phase-transition points $p_{c}$ estimated with MWPM [34]. (a) The two curves for $0 \leqslant q \leqslant 0.5$. Closeups of the regions near (b) $q=0$ and (c) $q=0.5$. (d) $p_{v}$ ( $q$ ) and its concave envelope. The dashed line indicates $q=0.46$ mentioned in the text. (e) $p_{c}$ and its concave envelope.

simple method could give some approximate location of the phase-transition point even for the diluted lattices, although we have not been able to directly derive their relationship.

The nonconcavity in the curve of $p_{c}$ was already reported in the previous work [34]. This was attributed to a finite-size effect [34] caused by large plaquettes that occupy approximately half of the primary square lattice in the range for the nonconcavity. Whether the occupation of a half of the lattice by a plaquette occurs depends on $q$ and $L$. The larger $q$, the larger $L$ we need to avoid such occupation. For the lattice size ( $L=128)$ used for our estimation of $p_{v}(q)$, such occupation does not occur for $q \leqslant 0.46$ at least [34]. Nevertheless, $p_{v}(q)$ exhibits the nonconcavity in $q \leqslant 0.46$ [Figs. 5(c) and 5(d)]. This fact implies that the nonconcavity of $p_{v}(q)$ is not due to the finite-size effect. For a fair comparison between $p_{v}(q)$ and $p_{c}$ in the range of large $q$, we need to clarify the origin of the nonconcavity of $p_{v}(q)$ or estimate $p_{c}$ for larger lattices, but this issue is out of the scope of the present study. 


\section{SUMMARY AND DISCUSSION}

We presented a possible approximate relation between frustration and the phase transition of Ising spin glasses on two-dimensional bond-diluted lattices at zero temperature. The relation is represented as the correspondence of points obtained by a simple method concerning frustration and the phase-transition points at zero temperature observed with varying the ratio of antiferromagnetic bonds. The method is based on extension of a previous one for lattices without dilution [27]. We calculate $v(q, p)$ defined by Eq. (7) using averaged quantities over diluted lattices or $v(p)$ defined by Eq. (5) for each diluted lattice. Both of the functions concern the derivative of the number of frustrated plaquettes with respect to the number of antiferromagnetic bonds in the lattice. This extension is more natural than another one previously proposed with an additional ansatz [33]. Motivated by the work for nondiluted lattices [27], where the condition that the obtained function is equal to unity leads to an approximate location of the phase-transition point, we applied the extended method to the diluted square lattice. Consequently, we found that both of the ways of extension typically give an almost identical result and that the obtained curve $p_{v}(q)$ as a function of $q$ is close to the correct phase boundary $p_{c}$ in the range $0 \leqslant q \leqslant 0.5$. For $q>0.4, p_{v}(q)$ exhibits the nonconcavity as $p_{c}$, but the origin of this behavior would be different from that of $p_{c}$. This issue is left as a future work.

The present result supports the validity of our method to approximately estimate the phase-transition points of twodimensional spin glasses at zero temperature on diluted lattices. The obtained solutions from our method are fairly accurate, even though what we did are only simple calculations. Hence our method has practical use for the first approximation of the transition points. The fairly good prediction with our method based only on frustration implies the importance of frustration on the ferromagnetic-spin-glass phase transition at zero temperature. It should be noted that our result dose not lead to the proposition of the geometryinduced transition at finite temperatures [16]. Our method does not evaluate thermal effects and is not associated with the phase transitions at finite temperatures. However, our method might be useful to investigate geometrical aspects of the phase transitions.

We have found the approximate correspondence between the solutions of our method and the phase-transition points at zero temperature, but its origin is not obvious. We need further investigation to clarify whether this agreement is reasonable or just an accident. We will examine other diluted lattices as the next task. In addition, an effect from Ising spins, which is not considered in our method at all, should be estimated to examine the possibility that our method could be associated with phase transitions. Investigation taking into account the nature of Ising spins in our method could find a link to a conventional approach to phase transitions in spin glasses, e.g., analysis of the domain-wall energy $[35,36]$.

\section{ACKNOWLEDGMENTS}

This research is partially supported by JSPS KAKENHI Grants No. 18H03303 and No. 19H01095, and the JSTCREST (Grant No. JPMJCR1402) for the Japan Science and Technology Agency.
[1] M. Mezard, G. Parisi, and M. Virasoro, Spin Glass Theory and Beyond, Lecture Notes in Physics (World Scientific, Singapore, 1987).

[2] K. H. Fischer and J. A. Hertz, Spin Glasses (Cambridge University Press, Cambridge, 1993).

[3] G. Parisi and F. Zamponi, Rev. Mod. Phys. 82, 789 (2010).

[4] P. Charbonneau, J. Kurchan, G. Parisi, P. Urbani, and F. Zamponi, Nat. Commun. 5, 3725 (2014).

[5] H. Nishimori, Statistical Physics of Spin Glasses and Information Processing: An Introduction (Oxford University Press, New York, 2001).

[6] M. Mezard and A. Montanari, Information, Physics, and Computation (Oxford University Press, Oxford, 2009).

[7] S. Morita, Y. Ozeki, and H. Nishimori, J. Phys. Soc. Jpn. 75, 014001 (2006).

[8] H. Nishimori, J. Stat. Phys. 126, 977 (2007).

[9] M. Ohzeki, H. Nishimori, and A. N. Berker, Phys. Rev. E 77, 061116 (2008).

[10] M. Ohzeki, Phys. Rev. E 79, 021129 (2009).

[11] M. Ohzeki and H. Nishimori, J. Phys. A 42, 332001 (2009).

[12] M. Ohzeki, Phys. Rev. A 85, 060301(R) (2012).

[13] M. Ohzeki and K. Fujii, Phys. Rev. E 86, 051121 (2012).

[14] M. Ohzeki, Phys. Rev. E 87, 012137 (2013).
[15] M. Ohzeki and J. L. Jacobsen, J. Phys. A: Math. Theor. 48, 095001 (2015).

[16] H. Nishimori, J. Phys. Soc. Jpn. 55, 3305 (1986).

[17] F. P. Toldin, A. Pelissetto, and E. Vicari, J. Stat. Phys. 135, 1039 (2009).

[18] A. Honecker, M. Picco, and P. Pujol, Phys. Rev. Lett. 87, 047201 (2001).

[19] S. L. A. de Queiroz, Phys. Rev. B 73, 064410 (2006).

[20] M. Achilles, J. Bendisch, and H. Trotha, Physica A 275, 178 (2000).

[21] C. Wang, J. Harrington, and J. Preskill, Ann. Phys. 303, 31 (2003).

[22] C. Amoruso and A. K. Hartmann, Phys. Rev. B 70, 134425 (2004).

[23] K. Fujii and Y. Tokunaga, Phys. Rev. A 86, 020303(R) (2012).

[24] N. Jinuntuya and J. Poulter, J. Stat. Mech. (2012) P01010.

[25] A. Kitaev, Ann. Phys. 303, 2 (2003).

[26] E. Dennis, A. Kitaev, A. Landahl, and J. Preskill, J. Math. Phys. 43, 4452 (2002).

[27] R. Miyazaki, J. Phys. Soc. Jpn. 82, 094001 (2013).

[28] G. Toulouse, Commun. Phys. 2, 115 (1977).

[29] S. Kirkpatrick, Phys. Rev. B 16, 4630 (1977).

[30] J. Vannimenus and G. Toulouse, J. Phys. C 10, L537 (1977). 
[31] A. N. Berker and S. Ostlund, J. Phys. C: Solid State Phys. 12, 4961 (1979).

[32] D. Sherrington and S. Kirkpatrick, Phys. Rev. Lett. 35, 1792 (1975).

[33] M. Ohzeki, Y. Kudo, and K. Tanaka, J. Phys. Soc. Jpn. 87, 015001 (2018).
[34] T. M. Stace, S. D. Barrett, and A. C. Doherty, Phys. Rev. Lett. 102, 200501 (2009); T. M. Stace and S. D. Barrett, Phys. Rev. A 81, 022317 (2010).

[35] C. Monthus and T. Garel, Phys. Rev. B 89, 184408 (2014).

[36] H. Khoshbakht and M. Weigel, Phys. Rev. B 97, 064410 (2018). 